\title{
OPINIONES DE LOS ESTUDIANTES DE ENFERMERÍA SOBRE EL APRENDIZAJE BASADO EN PROBLEMAS
}

\section{NURSING STUDENTS' OPINIONS ABOUT PROBLEM BASED LEARNING}

\author{
*Guerra Martín, Ma Dolores \\ *Profesora colaboradora. Departamento de Enfermería. Universidad de Sevilla.
}

Palabras clave: Opiniones de los estudiantes de enfermería, Aprendizaje basado en problemas, Método de aprendizaje

Keywords: Nursing Students' Opinions, Problem Based Learning, Learning Methods

\section{RESUMEN}

Introducción: Desde el curso 2005/2006 el Departamento de Enfermería (Universidad de Sevilla) ha fomentado la implantación de la estrategia de Aprendizaje Basado en Problemas (ABP), en la Diplomatura de Enfermería. En este trabajo presentamos la experiencia del $A B P$, en la asignatura de Administración de Servicios de Enfermería, en la Unidad Docente Valme, durante el curso 2007/2008.

Objetivo: Conocer las opiniones de los estudiantes sobre el ABP.

Método: Hemos realizado dos cuestionarios de elaboración propia para conocer las opiniones de los estudiantes sobre el trabajo de la tutora y sobre el trabajo en equipo. Se han realizado los cuestionarios al finalizar la asignatura, de una manera anónima y voluntaria.

Resultados: La muestra está compuesta por 53 estudiantes matriculados en la asignatura en la Unidad Docente Valme. Han contestado los cuestionarios: 51 estudiantes (96,22\%).

Media de la puntuación sobre la tutora (contestaciones $100 \%$ de la muestra): Puntualidad: 8,78 ; atención y escucha: 8,64; adecuación de los objetivos: 8,52; adecuación método enseñanza: 8,13 y adecuación de los recursos: 7,92. Alfa de Cronbach: 0,914.

Media de la puntuación sobre el trabajo en equipo (contestaciones 100\% de los alumno/as): Puntualidad: 7,76; participación constructiva: 7,72; Realización de las tareas: 7,90; respetan a los demás: 8,25 y capacidad de trabajo en equipo: 7,66 . Alfa de Cronbach: 0,793.

Conclusiones: Creemos que es conveniente establecer estrategias de mejora en todo el proceso y evaluación del ABP. Este método de aprendizaje es importante tenerlo en cuenta, a la hora de mejorar la calidad de la enseñanza. La puesta en marcha del sistema de créditos europeos favorece la implantación de este método de aprendizaje. 


\section{ABSTRACT}

Introduction: Since the academic year 2005/2006 the Department of Nursing at the University of Seville, Spain has promoted the implementation of the strategy of problem-based learning (PBL) in nursing degrees. The experience of $\mathrm{PBL}$ in the nursing services administration course at the Teaching Unit Valme, during 2007/2008 is presented in this study.

Objective: To know nursing students' opinions about PBL.

Method: We conducted two questionnaires specifically designed to find out the views of students about the tutor's work and teamwork. Questionnaires have been conducted at the end of the course in an anonymous and voluntary way.

Results: The sample consists of 53 students enrolled in the subject in the Valme Teaching Unit. They answered in the questionnaire: 51 students (96.22\%).

Average score of the tutor (responses $100 \%$ of the sample): Timeliness: 8.78; attention and listening: 8.64; adequacy of targets: 8.52; adequacy of teaching method: 8.13 and adequacy of resources: 7.92 . Cronbach alpha: 0.914 .

Average of team work (100 \% replies of the students): Timeliness: 7.76 ; constructive involvement: 7.72; Completing tasks: 7.90 ; respect for others: 8.25 and ability to work in teams: 7.66 . Cronbach alpha: 0.793.

Conclusions: We believe it is appropriate to establish improvement strategies throughout the process and evaluation of the PBL. This method of learning is important to bear in mind, so as to improve the quality of education. The launch of the European credit system facilitates the implementation of this method of learning.

\section{INTRODUCCIÓN}

Desde el curso 2005/2006, el Departamento de Enfermería (Universidad de Sevilla) ha fomentado la implantación de la estrategia de Aprendizaje Basado en Problemas (ABP), en la Diplomatura de Enfermería. En este trabajo presentamos la experiencia del $A B P$, en la asignatura de Administración de Servicios de Enfermería, durante el curso 2007/2008.

Para llevar a cabo el ABP presentamos un Proyecto de Innovación Docente denominado: "Utilización de la metodología de Aprendizaje Basado en Problemas con los alumnos/as de tercero de la Diplomatura de Enfermería", el cual fue aprobado y financiado dentro del Plan de Renovación de las Metodologías Docentes, correspondiente a la acción 5 de la Universidad de Sevilla.

El ABP es un método de enseñanza-aprendizaje, cuyo objetivo final es mejorar la calidad docente. Con este método de aprendizaje, los estudiantes pasan a ser sujetos activos de su aprendizaje, ya que construyen su conocimiento sobre la base de problemas de la vida real, que les van planteando los tutores/as y que los alumnos/as tienen que resolver. La misión de los tutores/as en este proceso de aprendizaje es la de facilitadores, catalizadores, ya que responsabilizan al alumno/a de su aprendizaje, aunque los tutores/as deben estimular el pensamiento crítico y si es necesario provocar la discusión y el debate.

La asignatura de Administración de Servicios de Enfermería es troncal y se imparte en el segundo cuatrimestre del tercer curso de la Diplomatura de Enfermería. Los créditos LRU (Ley Reforma Universitaria) asignados en el Plan de Ordenación Docente (POD) son cuatro para teoría y tres para preclínicas.

El total de créditos actual LRU para preclínicas, se distribuyó de la siguiente manera: 
Tutorías en grupo de ABP, con 1,4 créditos.

Seminarios de competencias en información, con 1,6 créditos.

El método del $A B P$ se ha llevado a cabo en el espacio y tiempo asignado para las preclínicas, estando detallado en programa de dicha asignatura. Existen dos Unidades Docentes pertenecientes al departamento de Enfermería, la Unidad Docente de Macarena y de Valme. La experiencia que vamos a detallar en este trabajo, se ha llevado a cabo con el alumnado de la Unidad Docente Valme.

El método del ABP aplicado en la asignatura Administración de Servicios está articulado en torno a problemas de la práctica gerencial y asistencial (ANEXOS I y II), lo cual produce una gran satisfacción entre el alumnado y una mayor implicación en el aprendizaje, ya que son problemas que pueden observar en sus prácticas clínicas.

Mediante la enseñanza virtual de la asignatura de Administración de Servicios de Enfermería (ANEXO III), los alumnos/as han podido acceder a toda la documentación elaborada por el tutor/a, la bibliografía, artículos, foro, correo, enlaces...En resumen, todo relacionado con el ABP y su desarrollo.

Los objetivos que nos planteamos con el ABP son los siguientes:

Capacitar a los alumnos/as a construir sus conocimientos sobre la base de problemas de la vida real.

Favorecer el aprendizaje activo del alumno/a.

Enseñar al alumno/a a aprender.

Integrar la teoría con la práctica cotidiana.

Favorecer el trabajo en equipo.

En cuanto al contenido y desarrollo de las tutorías de ABP, decir que se han llevado a cabo tres tutorías de ABP presenciales y tres tutorías no presenciales, en los tiempos asignados para las preclínicas de esta asignatura, en la modalidad de tutorías en grupo de aproximadamente diez alumnos/as para lo cual se han establecido 6 grupos. Debido a cómo se desarrolla y estructura el trabajo en el ABP, antes de comenzar la tutorías se advertía al alumnado participante, que no se permitía ningún cambio de grupo sin previa comunicación a la tutora y que los alumnos/as que empezaran en un grupo la 1a tutoría, debían continuar siendo los mismos en la 2a y $3 a$ tutoría.

En la primera tutoría (presencial) se entregó una copia de la primera parte del problema (ANEXO I) al grupo de alumnos/as los cuales realizaron después su lectura. Los alumnos/as analizaron el escenario sobre el que se presenta el problema, identificando lo que sabían o lo que creían saber del caso, poniendo en práctica el conocimiento previo (puede ser conocimiento adquirido a lo largo de la carrera, experiencias durante las prácticas, lecturas bibliográficas, experiencias personales o familiares.). El debate sobre el caso iba haciendo que los alumnos/as detectaran la necesidad de nuevos conocimientos, lo que da lugar a la búsqueda de información necesaria para resolverlo y así lograr los objetivos en cada tutoría. Los componentes del grupo mediante un trabajo de equipo, se repartían la búsqueda de información.

En la segunda tutoría (no presencial), realizaron las búsqueda de información necesaria en las distintas bases de datos (MEDLINE, CSIC, CINAHL, CUIDEN.), internet, revistas, libros, programa y bibliografía de la asignatura, consultas a expertos en hospitales, en 
centros de salud... El grupo elaboró una lista de información necesaria para solucionar el problema, así como los conceptos básicos para conocer la materia.

En la tercera tutoría (presencial), en una primera parte organizaron los alumnos/as toda la documentación encontrada por cada uno de los miembros del equipo, del problema presentado en la primera tutoría, dándole significado a la información necesaria para concretar el problema, con una puesta en común del trabajo de equipo y coloquio. En una segunda parte, se les entregó la segunda parte del problema (ANEXO II) y los alumnos/as discutieron los nuevos escenarios que se les presentaban. Nuevamente los alumnos/as detectaron la necesidad de conocimientos, lo que dio lugar a la búsqueda de información necesaria para resolverlo, el grupo analizó la información recopilada y buscaron nuevas opciones y posibilidades para la resolución del problema, identificando los temas a estudiar y estableciendo una lista de tareas para la próxima tutoría.

En la cuarta tutoría (no presencial), realizaron las búsquedas de información necesarias en las distintas bases de datos (MEDLINE, CSIC, CINAHL, CUIDEN...), internet, revistas, libros, programa y bibliografía de la asignatura, consultas a expertos en hospitales, en centros de salud. El grupo elaboró una lista de información necesaria para solucionar el problema, así como los conceptos básicos para conocer la materia.

En la quinta tutoría (presencial), en una primera parte organizaron los alumnos/as toda la información trabajada del problema y realizaron una puesta en común de los resultados de la segunda parte del problema y coloquio. Posteriormente, reorganizaron el grupo de alumnos/as toda la información trabajada del problema ( 1 a y $2^{\text {a }}$ parte), desarrollando habilidades de alto nivel como la evaluación, la toma de decisiones y la síntesis y trataron de elaborar unas conclusiones, de sintetizar las ideas y crear conocimiento para poder retroalimentarse y alentar al descubrimiento. Al final de esta tercera tutoría, se reservó un tiempo para la evaluación del grupo y evaluación de la tutora.

Para la evolución y consecución de los objetivos propuestos es muy importante la evaluación del trabajo en equipo, ya que esta retroalimentación sirve de estímulo para la mejora personal y grupal. Tanto el grupo como la tutora evaluaron tres estructuras de interrelación: la relación del grupo con los objetivos, medios y aprendizaje; la relación de los diferentes miembros dentro del equipo; y por último, la relación de los miembros con la tutora.

En cuanto a la evaluación de las tutorías de ABP presenciales y no presenciales, descrita en el programa de la asignatura, la puntuación máxima que podían tener los estudiantes, era de 1,4 puntos.

Los criterios de evaluación de las tutorías presenciales, eran los siguientes:

ia tutoría en grupo: 0.3 puntos por la asistencia y participación.

2a tutoría en grupo: 0.3 puntos por la asistencia y participación.

3a tutoría en grupo: 0.3 puntos por la asistencia y participación.

Con respecto a los criterios de evaluación de las tutorías no presenciales, se valoró como máximo 0.5 puntos por la presentación del trabajo llevado a cabo en el $A B P$, valorándolo de la siguiente manera:

Presentación del trabajo: 0.1 puntos.

Capacidad de síntesis: 0 a 0.2 puntos. 
Adecuación del trabajo a la temática tratada: 0 a 0.2 puntos.

\section{OBJETIVOS}

Conocer las opiniones de los estudiantes sobre el método del Aprendizaje Basado en Problemas.

\section{METODOLOGÍA}

Hemos realizado dos cuestionarios de elaboración propia para conocer las opiniones de los estudiantes, fueron realizados al finalizar la asignatura, de una manera anónima y voluntaria. Un cuestionario incluía las opiniones de los estudiantes sobre la tutora (ANEXO IV) y otro, sobre el trabajo en equipo (ANEXO V), ambos con cinco ítems cada uno y puntuable del uno al diez. El análisis de la información se llevó a cabo con el programa informático SSPS 15.0 .

\section{RESULTADOS}

\subsection{Resultados de los cuestionarios:}

Con respecto a los dos cuestionarios, los han contestado 51 estudiantes significando un $96,22 \%$ del total de alumnos/as matriculados. De esta muestra de 51 alumnos/as, contestaron según el género (GRÁFICO 1): 17 alumnos (33,33\%) y 34 alumnas $(66,67 \%)$. A continuación presentamos los resultados del $100 \%$ de la muestra, referidos a las medias por preguntas, de las opiniones de los estudiantes sobre la tutora (GRÁFICO 2) y sobre el trabajo en equipo (GRÁFICO 3).

\section{GRÁFICO 1}

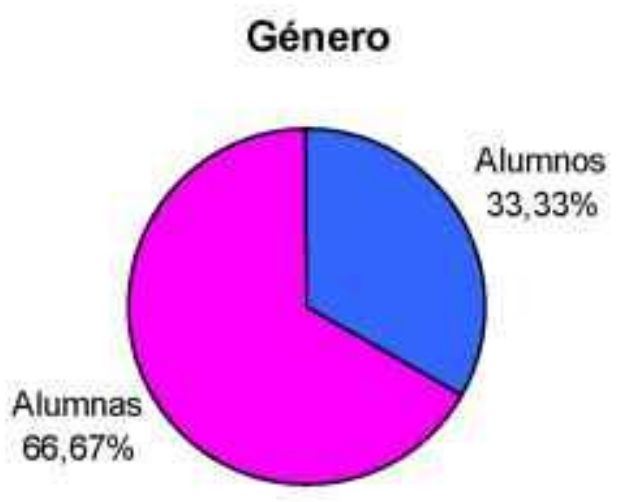




\section{GRÁFICO 2}

Resultados de opinión de los estudiantes sobre la tutora:

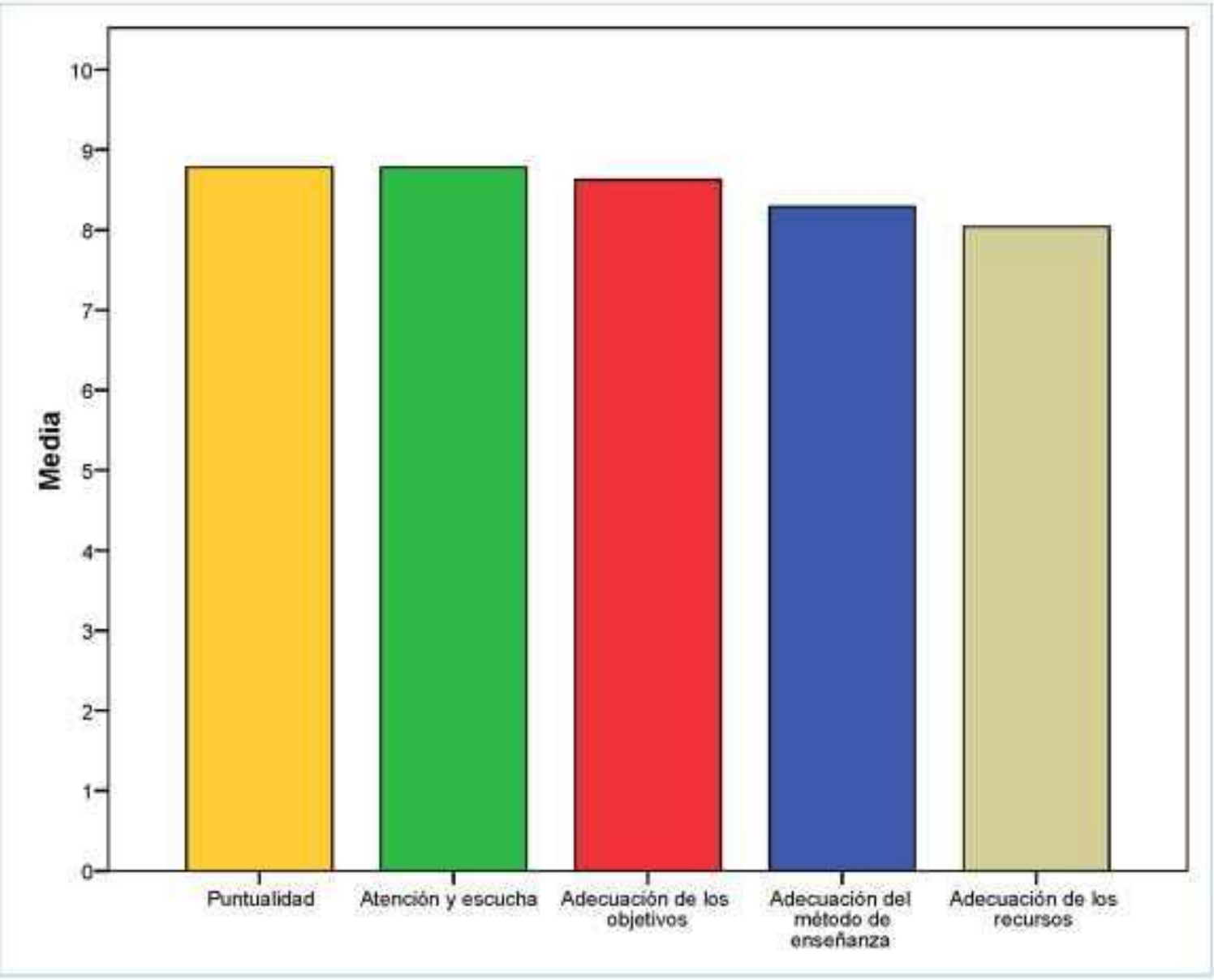




\section{GRÁFICO 3}

Resultados de opinión de los estudiantes sobre el trabajo en equipo:

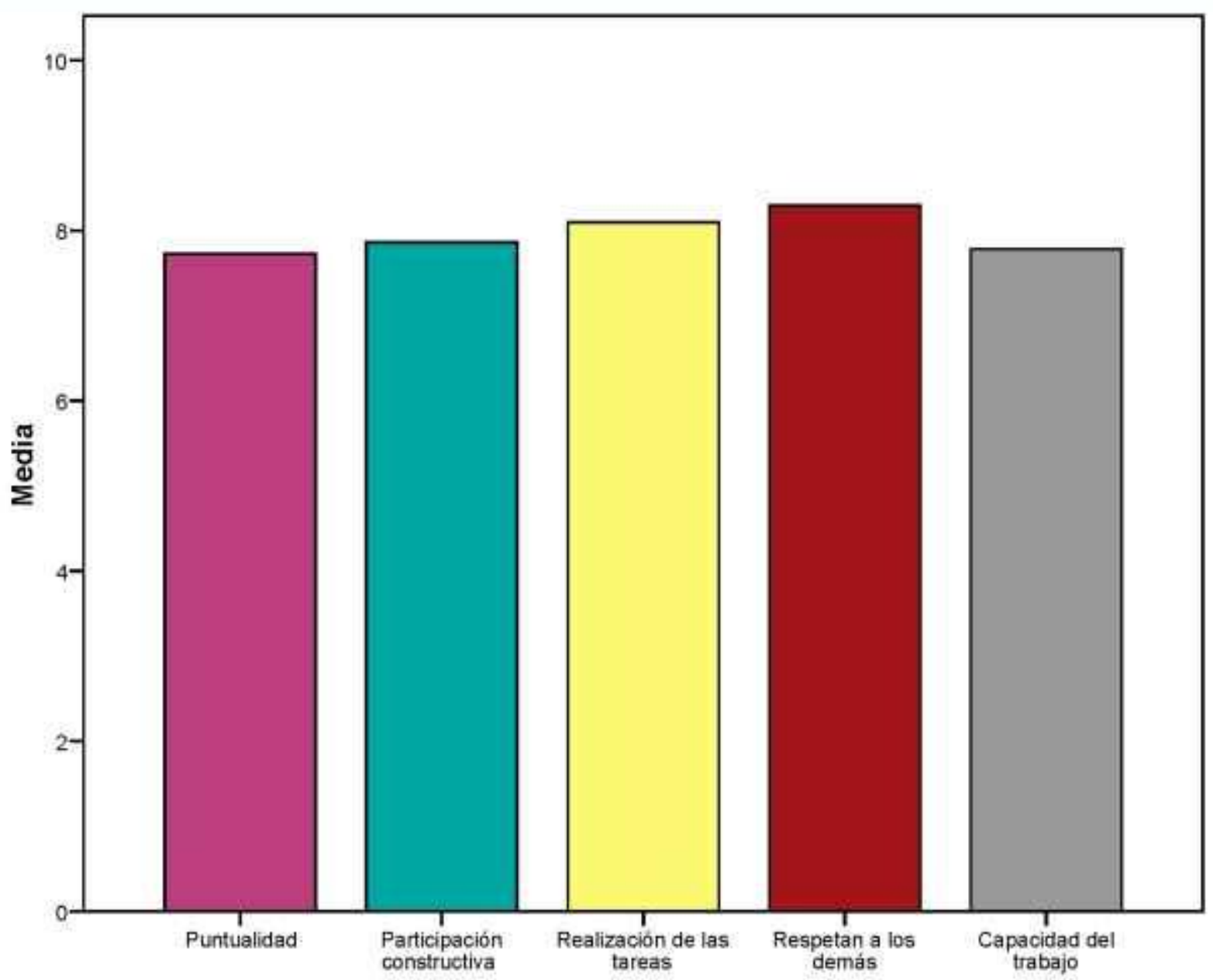

\subsubsection{Cuestionario sobre la tutora:}

1. Puntualidad: 8,78

2. Atención y escucha: 8,64

3. Adecuación de los objetivos: 8,52

4. Adecuación método enseñanza: 8,13

5. Adecuación de los recursos: 7,92

\subsubsection{Indice de fiabilidad de la tutora}

El indicador Alfa de Cronbach sirve para medir la fiabilidad de un test en escala de Likert. Si su valor está cercano a la unidad, se trata de un instrumento fiable que hace mediciones estables y consistentes, en nuestro caso está muy cercano a la unidad: 0,914 (ANEXO VII). 


\subsubsection{Cuestionario sobre el trabajo en equipo:}

1. Puntualidad: 7,76

2. Participación constructiva: 7,72

3. Realización de las tareas: 7,90

4. Respetan a los demás: 8,25

5. Capacidad de trabajo en equipo: 7,66

\subsection{3. Índice de fiabilidad del alumno}

Alfa de Cronbach: En este caso la fiabilidad está cercana a la unidad: 0,793 (ANEXO VIII), por lo que también se trata de un instrumento fiable.

\section{CONCLUSIONES}

El método de ABP es una herramienta metodológica de enseñanza innovadora que favorece el aprendizaje activo del alumnado. Con este método se pretende integrar la teoría y la práctica mediante el trabajo en equipo, con el objetivo de mejorar la calidad de la enseñanza.

El método del ABP aplicado en esta asignatura, mediante tutorías articuladas en torno a un problema de la práctica asistencial, produce una gran satisfacción entre el alumnado, ya que en sus prácticas clínicas se pueden encontrar con problemas similares. Por otro lado produce también, una mayor implicación del alumnado en su proceso de aprendizaje, viéndose esto refrendado por la alta asistencia y participación de los estudiantes en las tutorías.

La evaluación de los estudiantes se ha planteado en este curso de una manera complementaria, para conocer los tutores/as sus opiniones y poder mejorar las deficiencias detectadas. Los resultados que hasta ahora hemos analizado son satisfactorios, aunque creemos que es conveniente establecer estrategias de mejora en todo el proceso y evaluación del $A B P$, en los próximos cursos.

En los resultados del cuestionario de opinión sobre la docencia (ANEXO VI y GRÁFICO 4), que ha realizado a los estudiantes la Universidad se Sevilla, no encontramos discrepancias con los resultados obtenidos en nuestro trabajo. La mayoría de los alumnos/as refiere que se vinculan los contenidos de la asignatura con aspectos prácticos de la misma y que están satisfechos con la labor docente de la profesora.

En el curso 2008-2009 nos hemos planteado, realizar el ABP no solamente en la asignatura de Administración de Servicios de Enfermería, queremos integrarlo con otras asignaturas de tercer curso de la diplomatura de Enfermería, en concreto con Enfermería Geriátrica, Enfermería Médico Quirúrgica y Enfermería Psiquiátrica y de Salud Mental.

Por último decir, que este método de aprendizaje es importante tenerlo en cuenta, a la hora de mejorar la calidad de la enseñanza y que la puesta en marcha del sistema de créditos europeos favorece la implantación de este método de enseñanza. 


\section{BIBLIOGRAFÍA}

- Branda, L. Aprendizaje basado en problemas, centrado en el estudiante, orientado a la comunidad. En: Aportes para un cambio curricular en Argentina 2001. Jornadas de Cambio Curricular de la Facultad de Medicina de la Universidad de Buenos Aires. Buenos Aires: Organización Panamericana de la Salud. 2001: 79-101.

- Branda, L. A. "Innovaciones Educativas en Enfermería." Revista ROL de Enfermería. 2001, 24(4): 309-12.

- Branda, L. A. El aprendizaje basado en problemas en la formación en ciencias de la salud. In A. Alameda Cuesta (Ed.), El aprendizaje basado en problemas: Una herramienta para toda la vida. Agencia Laín Entralgo. Madrid, 2004.

- Colén, M. T. "La evaluación de los procesos de enseñanza y aprendizaje a través del portafolios. ¿Una moda o un proceso de evaluación que favorece el cambio en las aulas universitarias?" Comunicación presentada en V Jornades sobre Docencia Universitaria. L'avaluació dels aprenentatges de l'alumnat. Intercanvi d'experiències d'innovació. Barcelona, 2002.

- Cónsul Giribet, M., \& Montenegro Marchante, L. Cambio de paradigma docente: EUE "Vall d'Hebron": Una experiencia innovadora centrada en el aprendizaje basado en problemas (ABP). Revista ROL de enfermería. 2006, 29(10): 15-20.

- Dochy, F., Segers, M., y Dierick, S. "Nuevas Vías de Aprendizaje y Enseñanza y sus Consecuencias: una Nueva Era de Evaluación". Boletín de la Red Estatal de Docencia Universitaria. 2002, 2(2): 13-31.

- Font, A. "Una experiencia de autoevaluación y evaluación negociada en un contexto de aprendizaje basado en problemas (ABP)". Revista de la Red Estatal de Docencia Universitaria. 2003, 3(2): 100-112.

- Guerra, M. D. "Desarrollo de Aprendizaje Basado en Problemas en las preclínicas". Revista de acceso abierto de la Universidad de Murcia RED-U. 2007. http://www.um. es/ojs/index. php/actas-redu/index.

- Molina, J. A., García, A., Pedraz, A., y Antón, M. V. "Aprendizaje basado en problemas: una alternativa al método tradicional". Revista de la Red Estatal de Docencia Universitaria. 2003, 3(2): 79-85.

- Pedraz, A., Antón, M. V. y García, A. "Observación de una tutoría de ABP dentro de la asignatura de legislación y ética profesional en enfermería". Revista de la Red Estatal de Docencia Universitaria. 2003, 3(2): 87-93.

- Plan De Renovación de Metodologías Docentes. Vicerrectorado de Docencia. Universidad de Sevilla, disponible en www.us.es.

- Salinas, A.; Morales, J. A. y Martínez P. "Satisfacción del estudiante con el profesor de la UAM Agronomía y Ciencias de la UAT ". [En línea] TURevista Digi.U@T mayo 2008. Vol. $2 \mathrm{~N}^{\circ}: .4$. www.turevista.uat.edu. $\mathrm{mx}$

- Sobrepere, G.; Segarra, V.; Alasino, R. y Rinaldi, A. El C.A.T.: Una experiencia de autoaprendizaje. Cuarta Jornada Sobre Aprendizaje Cooperativo, del grupo GIAC. Universitat Politècnica de Catalunya, UPC. 2004. ISBN: 84-688-2760-6.

- Tribuna Sanitaria. Colegio Oficial de Diplomados en Enfermería de Madrid. La Enfermería apuesta por una nueva metodología docente basada en "aprender a aprender". No 159 Mayo 2003. https://codem.es/Tribuna/hemeroteca/tribuna 159.pdf

- Vazquez, S.; Mateos, M. D.; Ruíz, E. y Casado, R. Formación para la acción en el Departamento de Enfermería: El Método del ABP en la enseñanza clínica. Revista de acceso abierto de la Universidad de Murcia RED-U. 2007. http://www.um. es/ojs/index. php/actas-redu/index 


\section{ANEXO I}

APRENDIZAJE BASADO EN PROBLEMAS (ABP)

\section{1'PARTE DEL PROBLEMA:}

Juana Gómez es una supervisora de una unidad de cuidados de medicina interna, tiene cuarenta años, lleva diecinueve años de ejercicio profesional y seis meses en el cargo de supervisión. Juana siempre ha trabajado en hospitales, los seis primeros años trabajó por distintos servicios, posteriormente estuvo diez años trabajando en una unidad de cuidados intensivos y cuando tuvo oportunidad de seleccionar su puesto de trabajo, hace tres años, se decidió por elegirlo en medicina interna porque le parecía menos estresante que cuidados intensivos.

Durante casi tres años que llevaba trabajando en esta unidad, no se daba cuenta de los problemas de la misma, ya que según ella "iba a trabajar y no me interesaba nada más", pero desde que está de supervisora se está dando cuenta de que el clima laboral no es muy favorable, ya que hay varias enfermeras que no se llevan bien, e incluso algunas no se hablan entre ellas.

En el tiempo que Juana lleva como supervisora, ha realizado un curso de administración sanitaria, donde se ha hablado de las técnicas de planificación sanitaria dentro de la planificación sanitaria, por lo que cree conveniente averiguar cual es la técnica de planificación más idónea para que le de una información más exacta sobre que problemas existen en su unidad y cuáles debe priorizar (jerarquización de prioridades) para empezar a mejorar el ambiente de trabajo, teniendo en cuenta que esta supervisora debe distribuir los recursos en razón de su mejor utilización y que los criterios a tener en cuenta para la priorización, son variados. 


\section{ANEXO II}

\section{2" PARTE DEL PROBLEMA:}

Juana Gómez una vez que ha conocido los problemas que había un su unidad de cuidados de medicina interna, ha comprobado que necesita motivar a sus enfermeras. Esta supervisora sabe que hay distintos autores que tratan el tema de la motivación y cree que debe tener en cuenta la teoría bifactorial de Herzberg.

La distribución del trabajo, en esta unidad está repartida de una forma "igualitaria" por tareas, ya que se identifican las actividades que hay que realizar en el turno, se reparten las tareas a realizar por cada una de ellas y se encargan de anotar los datos correspondientes a estas tareas en las historias. En esta unidad hay muchas bajas laborales y continúas peticiones de cambios a otras unidades

En esta unidad de cuidados de medicina interna hay una plantilla de veinte enfermeras y dieciocho auxiliares de enfermería. Los turnos de trabajo de mañana y tarde son de siete horas y diez horas el turno de noche, la rotación es la siguiente: siete tardes después un día de descanso, siete mañanas después tres días de descanso, cuatro noches y después siete descansos, por último, tres noches y tres días de descanso. Al año hacían algo más de 1500 horas. Tienen un total de 47 camas de esta unidad.

La anterior supervisora (que este año se jubila) llevaba quince años en el puesto, era muy extrovertida y dejaba mucha autonomía a la plantilla de enfermeras, tenía un estilo de liderazgo que daba mucha libertad, "dejaba hacer" a las enfermeras, por lo que a Juana le está costando mucho trabajo conseguir que se hagan responsables estas enfermeras de la planificación de cuidados de sus pacientes, por lo que se está formando sobre cómo debe realizar la asignación de cuidados.

Juana opina que no se les puede prestar unos servicios de calidad a los pacientes, existiendo un mal clima laboral entre las enfermeras y no existiendo una planificación de los cuidados a los pacientes que ingresan en esta unidad de medicina interna. 


\section{ANEXO III}

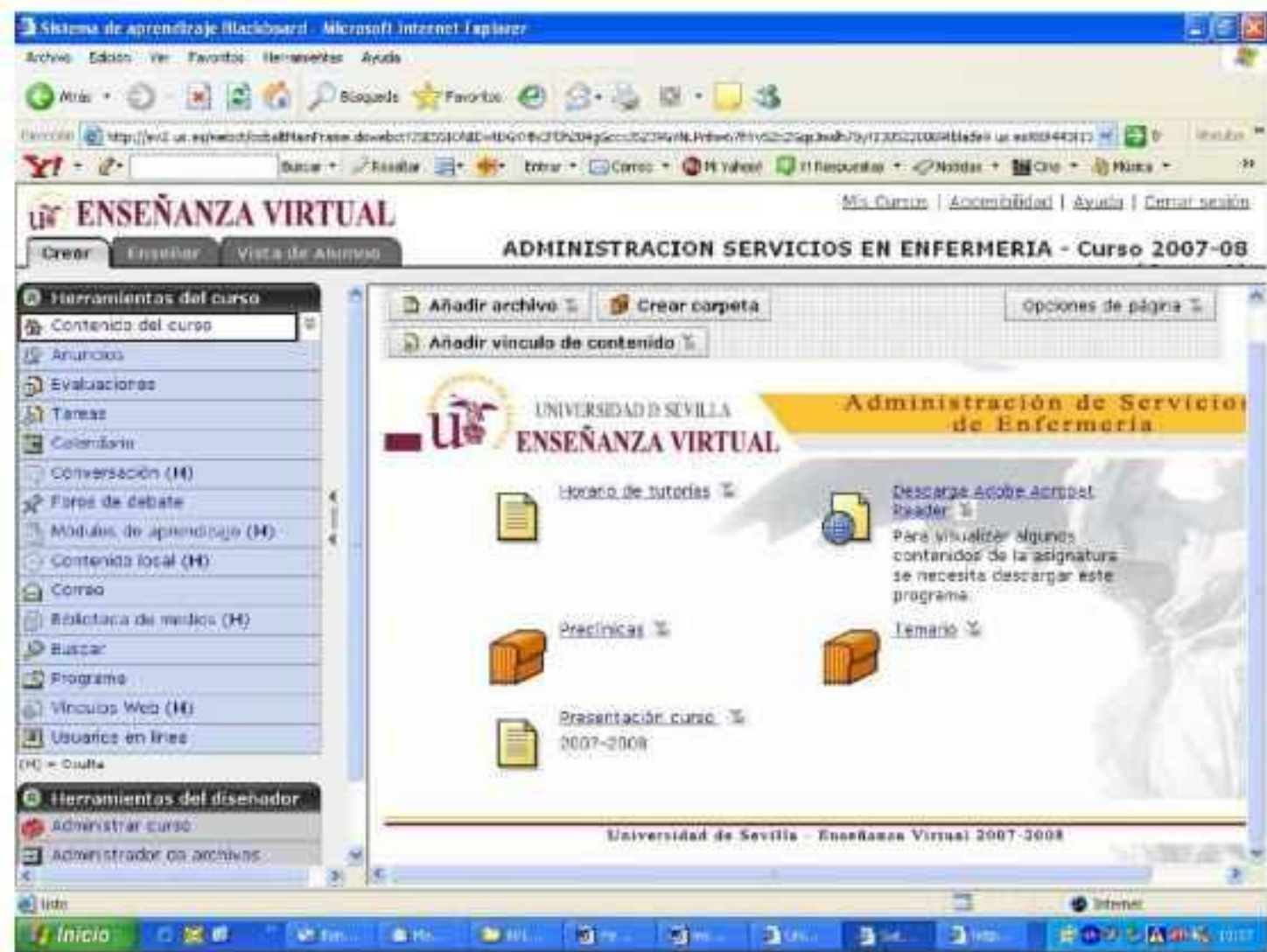

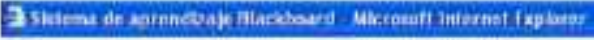

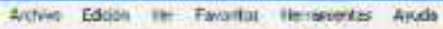

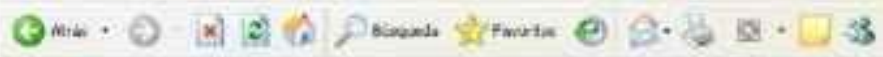

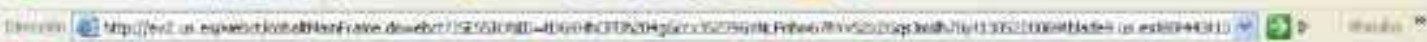

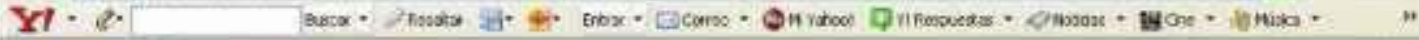

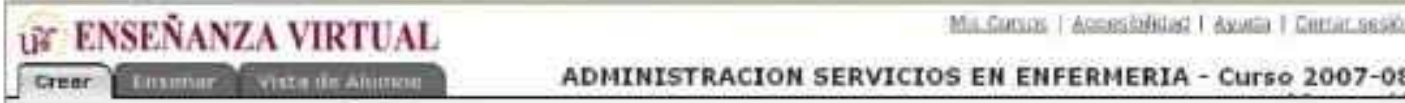

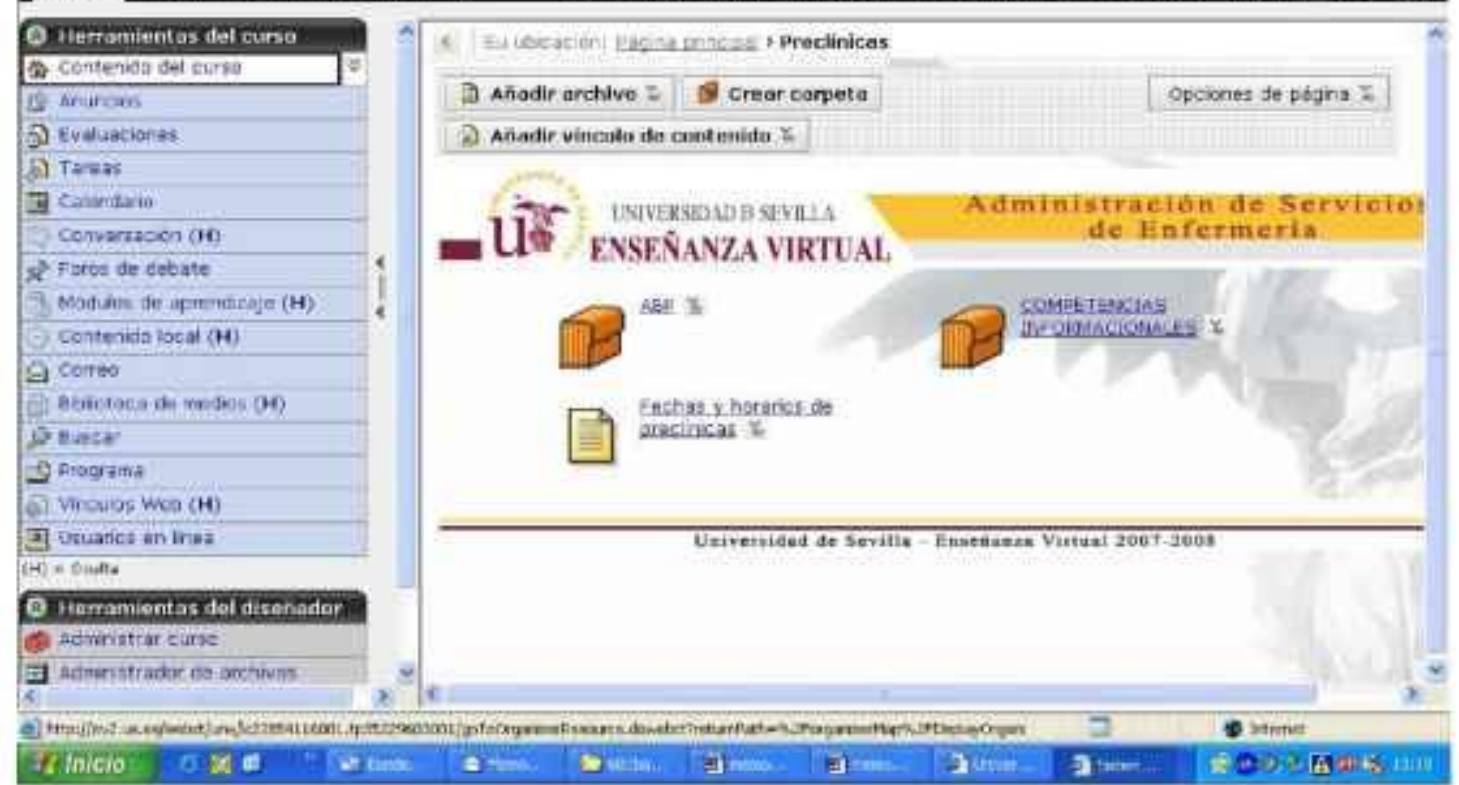




\section{ANEXO III (continuación)}

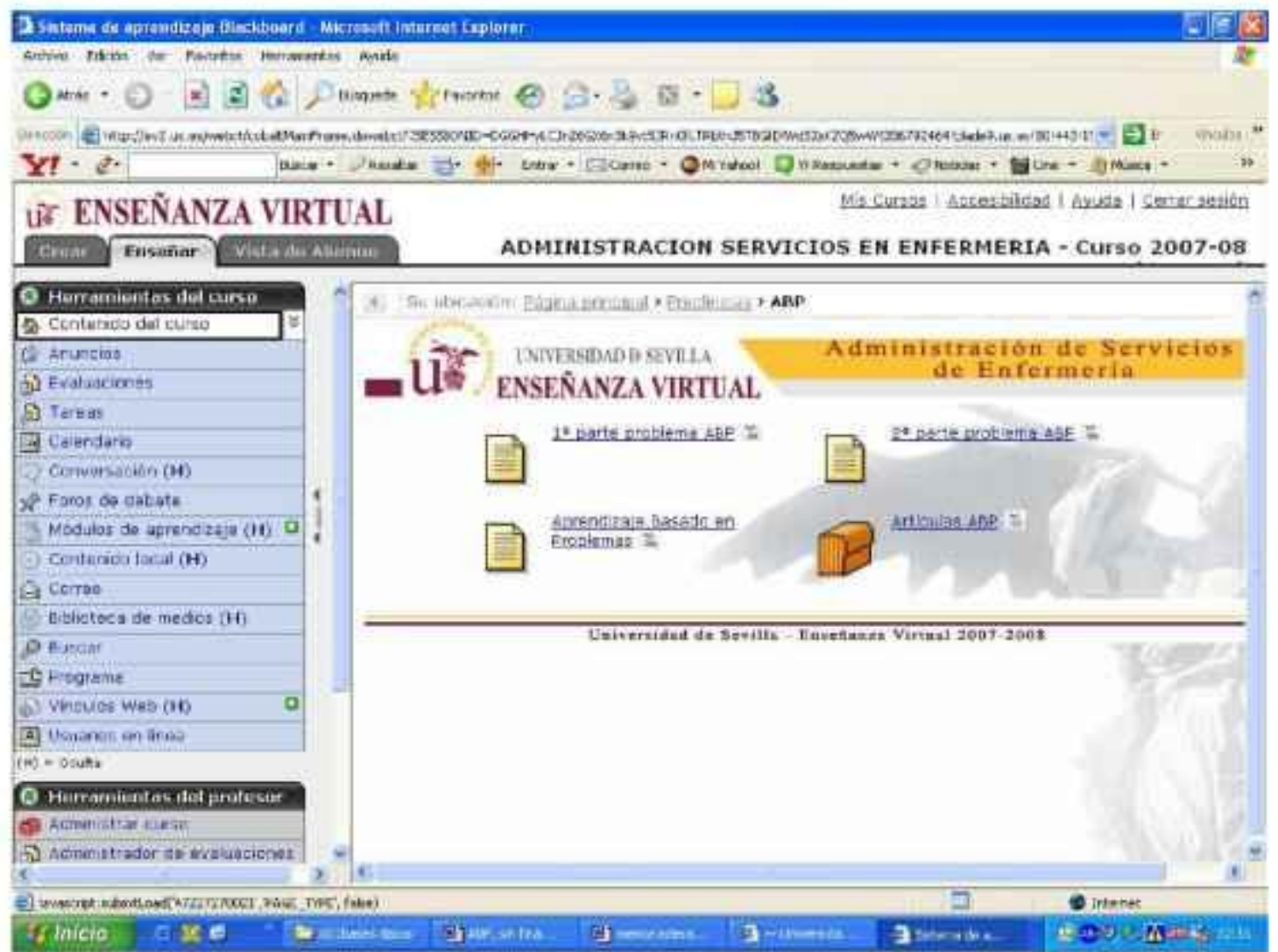




\section{ANEXO IV}

EVALUACIÓN DEL ALUMNADO DE LAS TUTORÍAS ABP

Evaluación de la tutora (Rodear la opción correcta)

Tutora:

\begin{tabular}{|l|l|cccccccccc|}
\hline & Puntualidad & 1 & 2 & 3 & 4 & 5 & 6 & 7 & 8 & 9 & 10 \\
& ABención y escucha & 1 & 2 & 3 & 4 & 5 & 6 & 7 & 8 & 9 & 10 \\
& Adecuación de los objetivos & 1 & 2 & 3 & 4 & 5 & 6 & 7 & 8 & 9 & 10 \\
& Adecuación método enseñanza & 1 & 2 & 3 & 4 & 5 & 6 & 7 & 8 & 9 & 10 \\
& Adecuación de los recursos & 1 & 2 & 3 & 4 & 5 & 6 & 7 & 8 & 9 & 10 \\
\hline
\end{tabular}




\section{ANEXO V}

EVALUACIÓN DEL ALUMNADO DE LAS TUTORÍAS ABP

Evaluación del trabajo en equipo (Rodear la opción correcta)

\begin{tabular}{|l|l|cccccccccc|}
\hline \multirow{4}{*}{ ABP } & Puntualidad & 1 & 2 & 3 & 4 & 5 & 6 & 7 & 8 & 9 & 10 \\
Participación constructiva & 1 & 2 & 3 & 4 & 5 & 6 & 7 & 8 & 9 & 10 \\
& Realización de las tareas & 1 & 2 & 3 & 4 & 5 & 6 & 7 & 8 & 9 & 10 \\
Respetan a los demás & 1 & 2 & 3 & 4 & 5 & 6 & 7 & 8 & 9 & 10 \\
Capacidad de trabajo en equipo & 1 & 2 & 3 & 4 & 5 & 6 & 7 & 8 & 9 & 10 \\
\hline
\end{tabular}




\section{ANEXO VI}

Resultados del cuestionario sobre la docencia, llevado a cabo por la Universidad de Sevilla:

El cuestionario era anónimo y voluntario pudiéndolos realizar los estudiantes al finalizar el curso. De los 53 estudiantes matriculados, los han respondido (muestra) 23 estudiantes significando un $43,39 \%$. Se podía acceder al cuestionario, mediante la enseñanza virtual, pudiendo responderlo el mismo alumno/a una sola vez. El cuestionario tenía diez preguntas, las cuales podían puntuarse del uno al diez. A continuación se presenta el informe de las medias de cada una de las preguntas:

1. El Profesor estructura la docencia de forma clara y ordenada: 8,70

2. Imparte la docencia de forma clara y ordenada: 8,52

3. Vincula los contenidos de la asignatura con aspectos prácticos de la misma (los relaciona con el ejercicio profesional, con la práctica, con aspectos de la vida,..): 8,52

4. El profesor ha intentado motivarme con esta asignatura: 8,39

5. Este profesor fomenta la participación del alumnado en su docencia: 8,09

6. Responde con claridad a las preguntas que realizan los estudiantes: 8,39

7. Las explicaciones del profesor son fácilmente comprensibles: 8,22

8. La evaluación se corresponde con los contenidos de la asignatura: 8,77

9. Las preguntas de la evaluación están formuladas con claridad: 8,45

10. En general estoy satisfecho con la labor docente de este profesor: 8,65 


\section{ANEXO VII}

Escala: Índice de fiabilidad de la tutora

Resumen del procesamiento de los casos

\begin{tabular}{|ll|r|r|}
\hline & \multicolumn{1}{|c|}{$N$} & \multicolumn{1}{c|}{$\%$} \\
\hline Casos & Válidos & 51 & 100,0 \\
& Excluidos(a) & 0 & 0 \\
& Total & 51 & 100,0 \\
\hline
\end{tabular}

Estadisticos de fiabilidad.

\begin{tabular}{|c|c|c|}
\hline & $\begin{array}{c}\text { Alfa de } \\
\text { Cronbach } \\
\text { basada en los } \\
\text { elementos } \\
\text { tipificados }\end{array}$ & $\begin{array}{c}\text { N de } \\
\text { elementos }\end{array}$ \\
\hline Cronbach & .914 & \\
\hline 914 & \\
\hline
\end{tabular}

Estadísticos de resumen de los elementos

\begin{tabular}{|l|r|r|r|r|r|r|r|}
\hline & Media & Minimo & Máximo & Rango & $\begin{array}{c}\text { Máximo/mi } \\
\text { nimo }\end{array}$ & $\begin{array}{c}\text { N de } \\
\text { Varianza }\end{array}$ & elementos \\
\hline Medias de los elementos & 7,953 & 7,725 & 8,294 &, 569 & 1,074 &, 056 & 5 \\
\hline
\end{tabular}




\section{ANEXO VIII}

\section{Escala: Índice de fiabilidad del alumno}

\section{Resumen del procesamiento de los casos}

\begin{tabular}{|ll|r|r|}
\hline & \multicolumn{1}{|c|}{ N } & \multicolumn{1}{|c|}{$\%$} \\
\hline Casos & Válidos & 51 & 100,0 \\
& Excluidos(a) & 0 &, 0 \\
& Total & 51 & 100,0 \\
\hline
\end{tabular}

\section{Estadisticos de fiabilidad}

\begin{tabular}{|r|c|c|}
\hline & $\begin{array}{c}\text { Alfa de } \\
\text { Cronbach } \\
\text { basada en los } \\
\text { elementos } \\
\text { tipificados }\end{array}$ & $\begin{array}{c}\mathrm{N} \text { de } \\
\text { elementos }\end{array}$ \\
\hline $\begin{aligned} \text { Cronbach } \\
.793\end{aligned}$ &, 800 & 5 \\
\hline
\end{tabular}

\section{Estadísticos de resumen de los elementos}

\begin{tabular}{|l|r|r|r|r|r|r|}
\hline & Media & Minimo & Máximo & Rango & $\begin{array}{c}\text { Máximo/mi } \\
\text { nimo }\end{array}$ & $\begin{array}{c}\text { N de } \\
\text { Varianza } \\
\text { elementos }\end{array}$ \\
\hline Medias de los elementos & 8,506 & 8,039 & 8,784 &, 745 & 1,093 &, 108 \\
\hline
\end{tabular}

\section{ISSN 1695-6141}

